\title{
EFECTO DE UN FOSFATO TÉRMICO SOBRE EL CRECIMIENTO Y PRODUCCIÓN DE FRIJOL (Phaseolus vulgaris L.) CV ICA CERINZA
}

\section{EFFECT OF THERMAL PHOSPHATE ON GROWTH AND PRODUCTION OF COMMON BEAN (Phaseolus vulgaris L.) CV 'ICA CERINZA'}

\author{
Wilmer Alejandro Quintana-Blanco ${ }^{1}$, Elberth Hernando Pinzón Sandoval ${ }^{2}$, David Fernando Torres ${ }^{3}$
}

\begin{abstract}
${ }^{1}$ Ing. Agrónomo, Investigador Grupo de Investigaciones Agrícolas. Universidad Pedagógica y Tecnológica de Colombia, Tunja-Boyacá, Av. Central del Norte, e-mail: wilquintana61@hotmail.com; ${ }^{2}$ Ing. Agrónomo, M.Sc. Fisiología Vegetal, Grupo de Investigaciones Agrícolas. Universidad Pedagógica y Tecnológica de Colombia, e-mail: elberth02@gmail.com; ${ }^{3}$ Ing. Agrónomo, M.Sc. Fisiología Vegetal, Estudiante Maestría en Ciencias Agrarias. Universidad Pedagógica y Tecnológica de Colombia, Av. Central del Norte, Tunja-Boyacá, email: mollisol89@hotmail.com
\end{abstract}

Rev. U.D.C.A Act. \& Div. Cient. 20(1): 51-59, Enero-Junio, 2017

\section{RESUMEN}

El fríjol es la leguminosa más importante del mundo por su aporte a la seguridad alimentaria y por el alto contenido de proteínas y de minerales esenciales. En américa latina y especialmente en Colombia, el cultivo de fríjol es importante en la economía campesina, pero, en la actualidad, la producción no satisface la demanda, haciendo necesario importar este producto. En la zona ecuatorial y subecuatorial, los suelos, en su mayoría, presentan condiciones ácidas, que conlleva a una baja fertilidad y limitación en la toma de elementos, como el fósforo. Por esto, el uso de fuentes de fósforo no convencionales representa una alternativa en la disponibilidad de este elemento, que mejore la dinámica nutricional y así generar un buen crecimiento y producción de los cultivos. El objetivo de esta investigación fue evaluar el efecto de un fosfato térmico sobre el crecimiento y el rendimiento del cultivar de fríjol 'ICA Cerinza', bajo condiciones agroecológicas, del municipio de Tunja-Boyacá. Se empleó un diseño completamente al azar, con cuatro tratamientos correspondientes a dosis de fosfato térmico 0, 300, 600 y $900 \mathrm{~kg} \mathrm{ha}^{-1}$, con cuatro replicaciones. Se evaluó el contenido total de clorofila, área foliar, peso seco de órganos, numero de vainas por planta y granos por vaina, peso de cien granos, rendimiento y contenido foliar de fósforo. Se presentaron diferencias estadísticas entre tratamientos $(P \leq 0,05)$, para las variables de crecimiento y el número de vainas por planta. La aplicación de $600 \mathrm{~kg} \mathrm{ha}^{-1}$ de fosfato térmico mostró los mejores resultados, posiblemente, debido a las características del producto, tales como solubilidad y composición, siendo una alternativa en suelos de condiciones moderadamente ácidas.
Palabras clave: Fósforo, acidez intercambiable, fosfato térmi$\mathrm{co}$, nutrición mineral.

\section{SUMMARY}

The common bean is the world's most important legume for their contribution to food security. In Latin America and especially in Colombia, the bean crop is important in the rural economy, but currently the production does not meet demand, necessitating import this product. In tropical and subtropical soils areas mostly present conditions exchangeable acidity, it leads to low fertility and limitation in making elements such as phosphorus, so the use of unconventional sources of phosphorus, may represent an alternative in the availability of this element that improves the nutritional dynamics and generate good growth and crop production. Therefore, the objective of this research was to evaluate the effect of a thermal phosphate on growth and productivity of bean cv. 'ICA Cerinza' under agro-ecological conditions of the municipality of Tunja-Boyacá. Design was used completely randomized with four treatments corresponding to doses of thermal phosphate $0,300,600$ and $900 \mathrm{~kg} \mathrm{ha}^{-1}$ with four replications. The total chlorophyll content, leaf area, dry weight of organs, yield components and leaf phosphorus content was evaluated. Statistical differences between treatments $(P \leq 0.05)$ for growth variables and the number of pods per plant were presented. Applying 600kg thermal phosphate $\mathrm{ha}^{-1}$ showed the best results, possibly because the product characteristics such as solubility and composition, being an alternative flooring moderately acidic conditions. 
Key words: Phosphorus, legume, exchangeable acidity, thermal phosphate, mineral nutrition.

\section{INTRODUCCIÓN}

El fríjol común (Phaseolus vulgaris L.) es la leguminosa alimenticia más importante para consumo directo en el mundo, por su alto contenido de proteínas y de minerales esenciales (Ulloa et al. 2011). Es cultivado por pequeños agricultores en América Latina y África, principalmente, en condiciones desfavorables y con mínimos insumos (Beebe et al. 2008). Según FENALCE (2015), el fríjol ha sido identificado por organismos nacionales e internacionales, como uno de los cultivos obligatorios para los programas de seguridad alimentaria. Los principales productores son América Latina y África, que aportan cerca del $50 \%$ de la producción mundial (Broughton et al. 2003).

En Colombia, el cultivo de fríjol es unas de las principales actividades de la economía campesina en varias regiones, especialmente, de la zona andina; de gran importancia, como generador de ingresos y empleo rural, pero que ha perdido competitividad frente a la producción de otros países, especialmente, por los altos costos de producción y bajos rendimientos (Arias et al. 2007). Según FENALCE (2015), el consumo anual de fríjol en Colombia es de 135.511t, con una producción aproximada de 101.497t, en un área de 94.441 ha, teniendo un déficit de producción de 34.014t para cubrir la demanda, el cual, es suplido por importaciones de países, como Perú, Estados Unidos, Ecuador y Bolivia.

El fósforo es un elemento esencial porque la planta no puede culminar su ciclo biológico en su ausencia, no puede ser sustituido por otro elemento y debe participar directamente en el metabolismo de las plantas (Fernández, 2007). Se encuentra disponible como ion fosfato y se absorbe, preferentemente, como $\mathrm{H}_{2} \mathrm{PO}_{4}{ }^{-}$en suelos, con un $\mathrm{pH}$ inferior a 7 y como anión divalente $\mathrm{HPO}_{4}{ }^{2-}$ en suelos, con un $\mathrm{pH}$ por encima de 7 (Azcón-Bieto \& Talón, 2013). Este elemento desempeña funciones estructurales en las macromoléculas, como los ácidos nucleicos y de transferencia de energía en los procesos metabólicos de biosíntesis y degradación. Permanece en su forma más altamente oxidada, ya que no son reducidos en la planta, lo que sí ocurre con los nitratos y los sulfatos (Marschner, 2012).

En zonas ecuatoriales y subecuatoriales de América Latina, África y Asia, existen grandes extensiones de suelos ácidos y de baja fertilidad natural, por presencia de altas concentraciones de elementos, como el aluminio (Zapata \& Roy, 2007). Esta condición genera la precipitación del fósforo, en forma de fosfatos de aluminio y tiene como resultado bajas concentraciones de P, disponible en el suelo. Esto ocasiona retraso en la floración, deficiente formación de órganos re- productivos, puede afectar la radiación interceptada por el cultivo, ya que el fósforo hace parte de enzimas fotosintéticas, como rubisco-pep-carboxilasa y de compuestos, tales como ATP, NADP, fosfolípidos, ADN, ARN e interviene en procesos, como la fotosíntesis, la absorción de iones, la síntesis de proteínas y compuestos orgánicos y la translocación de asimilados (Fernández, 2007).

En la actualidad, la mayoría de fertilizantes fosfóricos se deriva de la roca fosfórica, nombre genérico utilizado para denominar todos los materiales que contienen fosfatos, con algunas impurezas, como sílice, minerales arcillosos, calcita, dolomita y óxidos hidratados de hierro y de aluminio (Zapata \& Roy, 2007). Las rocas fosfóricas son un recurso no renovable, por tanto, se prevé que en los próximos 50 años las fuentes de fósforo se agotarán, lo que conllevaría a un gran problema en la producción de los cultivos, reflejado en la seguridad alimentaria mundial (Cordell et al. 2009; Gilbert, 2009).

Existe la posibilidad de obtención de un derivado de la roca fosfórica con propiedades de mayor disponibilidad en condiciones de acidez, denominado fosfato térmico, que es un fertilizante fosfórico, obtenido a través del tratamiento térmico a $1600^{\circ} \mathrm{C}$, que rompe la estructura rígida de la roca fosfórica, produciendo un material con una solubilidad intermedia, lo cual, mejora la disponibilidad a corto y largo plazo y ofrece a la planta un mayor porcentaje de P, en forma asimilable durante todo su ciclo fenológico (Fernández \& Noguera, 2003). El fosfato térmico contiene, además, concentraciones de calcio, de magnesio y de silicio, proveniente de los materiales que componen la roca fosfórica, que estimulan la absorción de fósforo, principalmente, en suelos de condición ácida (Fernández, 2007).

El objetivo de la investigación fue evaluar el efecto de tres dosis de un fosfato térmico frente a un testigo sin aplicación en el crecimiento y en la producción de fríjol (Phaseolus vulgaris L.) cv. 'ICA Cerinza', bajo condiciones agroecológicas del municipio de Tunja, departamento de Boyacá.

\section{MATERIALES Y MÉTODOS}

El estudio, se realizó bajo condiciones de campo abierto, en la granja La María, de la Universidad Pedagógica y Tecnológica de Colombia, con sede en Tunja, Boyacá, con coordenadas $5^{\circ} 32^{\prime 2} 5^{\prime \prime} \mathrm{N} 73^{\circ} 21^{\prime} 41^{\prime \prime} \mathrm{O}$ y una altura de $2.691 \mathrm{msnm}$. Las condiciones climáticas durante el desarrollo del experimento fueron las siguientes: temperatura de $13,9^{\circ} \mathrm{C}$, humedad relativa del $70 \%$ y una precipitación de $81,7 \mathrm{~mm}$ durante los meses de octubre del 2015 y enero de 2016.

Se utilizó un diseño completamente al azar con cuatro tratamientos: T1: Testigo sin aplicación; T2: 300 $\mathrm{kg} \mathrm{ha}^{-1}$; T3: 
$600 \mathrm{kgha}^{-1}$ y T4: $900 \mathrm{~kg} \mathrm{ha}^{-1}$ del producto comercial T-Fos ${ }^{\circledR}$ (fosfato térmico), de la empresa Cales y Derivados Calcáreos Río Claro Naranjo y Compañía S.C.A., estos determinados, según las condiciones del suelo (Tabla 1). El producto comercial tiene una composición garantizada de $\mathrm{P}_{2} \mathrm{O}_{5} 20 \%$; $\mathrm{CaO} 28 \% ; \mathrm{MgO}$ 8,5\%; $\mathrm{SiO}_{2}$ 15\%. Cada tratamiento se replicó cuatro veces, para un total de 16 unidades experimentales (UE). Las UE fueron parcelas con dimensiones $1,4 \times 2 \mathrm{~m}$, para un área de $2,8 \mathrm{~m}^{2} /$ parcela. La siembra, se hizo con una distancia entre surcos de $0,6 \mathrm{~m}$ y entre plantas de $0,3 \mathrm{~m}$, para una densidad de 55.555 plantas ha ${ }^{-1}$. Se colocaron dos granos por sitio, para luego realizar un raleo y tener un total de 18 plantas por UE.

Tabla 1. Propiedades físico-químicas del suelo utilizado en el experimento.

\begin{tabular}{|c|c|c|}
\hline Propiedad & Valor & Interpretación \\
\hline $\mathrm{pH}$ & 5,53 & Moderadamente. Ácido (Castro, 1998) \\
\hline $\mathrm{M} . \mathrm{O}(\%)$ & 0,99 & Baja \\
\hline $\mathrm{P} \mathrm{Bray} \mathrm{II}(\mathrm{ppm})$ & 22,7 & Alto \\
\hline $\mathrm{Ca}\left(\mathrm{cmol} \mathrm{kg}^{-1}\right)$ & 11,35 & Alto \\
\hline $\mathrm{Mg}\left(\mathrm{cmol} \mathrm{kg}^{-1}\right)$ & 3,6 & Alto \\
\hline $\mathrm{K}\left(\mathrm{cmol} \mathrm{kg}^{-1}\right)$ & 1,88 & Alto \\
\hline $\mathrm{Na}\left(\mathrm{cmol} \mathrm{kg}^{-1}\right)$ & 0,75 & Medio \\
\hline $\left.\mathrm{CE}(\mathrm{dS} \mathrm{m})^{-1}\right)$ & 0,63 & Baja \\
\hline Textura & A: $28,8 \mathrm{Ar}: 26 \mathrm{~L}: 45,12$ & Franca \\
\hline
\end{tabular}

Para la medición de las variables, se seleccionaron 10 plantas de los surcos centrales, con el fin de evitar el efecto de borde. Se utilizó semilla de fríjol tipo arbustivo cultivar 'ICA Cerinza', de buena adaptación en la zona. Antes de la siembra, se realizó un análisis fisicoquímico del suelo (Tabla 1). La aplicación del fosfato térmico, se hizo al momento de la siembra. La fertilización, se efectuó de acuerdo a los resultados del análisis de suelos un mes después de la siembra, utilizando Urea, DAP, Cloruro de Potasio y Micronfos. Se aplicó riego por aspersión, según necesidad del cultivo y aplicación de control fitosanitario, con base en monitoreo, aplicando productos para el control de antracnosis y minadores.

Las variables fisiológicas y de crecimiento evaluadas fueron: clorofila total SPAD (con un clorofilómetro Minolta SPAD 502 plus), tomando por planta un total de 10 mediciones en hojas del tercio central; grosor de hoja $\mathrm{mm}$ (por medio de un calibrador digital Mitutoyo precisión $\pm 0,05 \mathrm{~mm}$ ); área foliar $\mathrm{cm}^{2}$ (con un medidor CI 202, Bio-Science Inc.); pesos frescos y secos $\mathrm{g}$ (en una balanza electrónica Acculab VIC 612 de $0,01 \mathrm{~g}$ de precisión y secadas en una estufa de secado Memmert, a $70^{\circ} \mathrm{C}$, durante $48 \mathrm{~h}$ ); contenido de fósforo en tejido, por el método de calcinación a $600^{\circ} \mathrm{C}$; digestión ácida y valoración por espectrofotometría visible. En cuanto al rendimiento, se realizó la medición de variables, como: número de vainas por planta, número de granos por vaina, peso de 100 granos al $14 \%$ de humedad g, balanceado, con un medidor Motomco Moisture Meter modelo 919, del laboratorio de semillas de FENALCE y rendimiento en $\mathrm{kg} \mathrm{ha}^{-1}$. Los análisis de fósforo, se efectuaron en el laboratorio de suelos y aguas de la Facultad de Agronomía de la Universidad Nacional de Colombia, sede Bogotá. La medición de variables de crecimiento y de producción, se adelantaron en el laboratorio de fisiología vegetal de la UPTC.

Los datos obtenidos fueron sometidos a pruebas de normalidad y de homogeneidad de varianza, mediante las pruebas de Shapiro-Wilk y Levene, respectivamente. Comprobados los supuestos, se realizó análisis de varianza; las variables que mostraron diferencias estadísticas fueron sometidas a pruebas de comparación de medias de Tukey $(\mathrm{P} \leq 0,05)$. Los análisis se formalizaron con el programa estadístico SAS v.9.2e SAS (Institute Inc., Cary, NC).

\section{RESULTADOS Y DISCUSION}

El contenido de clorofila presentó diferencias estadísticas $(P \leq 0,05)$ entre tratamientos, desde la aparición de hojas verdaderas hasta llenado de grano (Figura 1). El tratamiento que presentó valores más altos en el contenido de clorofila fue el de $600 \mathrm{~kg} \mathrm{ha}^{-1}$ de fosfato térmico, con un valor de $53,5 \pm 0,96$ unidades SPAD, en la semana 14. Según Shekari et al. (2015), el contenido de clorofila está directamente ligado a la tasa fotosintética, en la cual, el fósforo tiene una 
participación directa en la transferencia de energía y el metabolismo de los hidratos de carbono, en los cloroplastos. Por otra parte, los procesos fotosintéticos son dependientes de los precursores, como el fosfato inorgánico o intermedios fosforilados, tales como ADP, ATP, NADPH, esenciales para la transferencia de energía (Singh et al. 2013).

Sumado a lo anterior, el fosfato térmico utilizado en la investigación también presenta un porcentaje de silicio y de magnesio; el silicio contribuye al aumento del contenido de clorofila en unidades SPAD (Soil Plant Analysis Development), debido a que está presente en altas concentraciones por unidad de área de tejido foliar, representando un impacto positivo en la tolerancia de la planta, a bajos o altos niveles de luz, haciendo más eficiente su uso por parte de los pigmentos fotosintéticos, como la clorofila (Adatia \& Besford, 1986); por otra parte, el magnesio aportado por el fosfato térmico interviene en varias funciones vitales para la planta, como la formación de clorofila (Cakmak \& Kirkby, 2008).

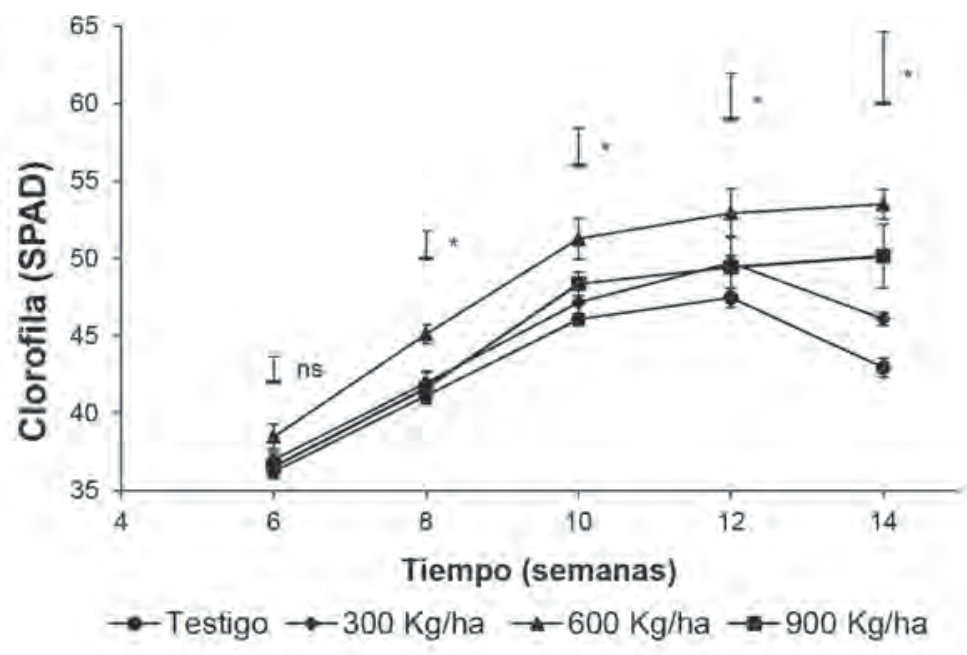

Figura 1. Comportamiento de la clorofila total en fríjol (Phaseolus vulgaris L.) cv 'ICA Cerinza', sometido a diferentes dosis de fosfato térmico. Barras verticales en los tratamientos indican error estándar $(n=4)$. Barras sobre los puntos de muestreo indica la diferencia mínima significativa en cada punto de muestreo, según la prueba de Tukey $(P \leq 0,05)$, ns: no existen diferencia estadística, *: diferencias significativas.

Según Cakmak et al. (1994), en fríjol y en trigo, cuando existe deficiencia de $\mathrm{Mg}$, se inhibe la concentración de clorofila, ya que el $\mathrm{Mg}$ es parte fundamental de la estructura de la misma. Los resultados encontrados en el presente estudio muestran que la aplicación de dosis crecientes del fosfato térmico hasta $600 \mathrm{~kg} \mathrm{ha}^{-1}$ aumentan el contenido de clorofila en el cultivo de fríjol cv 'ICA Cerinza'; sin embargo, el tratamiento de $900 \mathrm{~kg} \mathrm{ha}^{-1}$ mostró un valor inferior, posiblemente, por exceso del contenido de fósforo en la solución del suelo, ocasionando un antagonismo con aniones, como el $\mathrm{NO}_{3}{ }^{-}$, lo que pudo causar una disminución leve en el indicador de clorofila total cuantificada, mediante el método no destructivo SPAD, que relaciona el contenido de nitrógeno, como componente de las clorofilas. Singh \& Reddy (2015) afirman que la capacidad fotosintética y, por ende, la clorofila, están relacionadas directamente con el contenido de fósforo y de nitrógeno en la planta.
El peso seco de órganos mostró diferencias significativas entre tratamientos $(P \leq 0,05)$. El mayor valor de raíz, de tallo, de hojas y de vainas lo presentó la dosis de $600 \mathrm{~kg} \mathrm{ha}^{-1}$ de fosfato térmico, con valores de $1,09 \pm 0,25 \mathrm{~g}, 15,79 \pm 1,28 \mathrm{~g}$, $21,18 \pm 1,76 \mathrm{~g}$ y $63,81 \pm 3,60 \mathrm{~g}$, respectivamente (Figura 2 ). El tratamiento testigo fue el que mostró menor valor en todos los casos y la dosis de $900 \mathrm{~kg} \mathrm{ha}^{-1}$ mostró una disminución frente al tratamiento de $600 \mathrm{~kg} \mathrm{ha}^{-1}$, con diferencias significativas en peso seco de hojas y de vainas. La acumulación de materia seca por la planta está directamente relacionada con el suministro de nutrientes, siendo la masa seca un indicador de la eficiencia en la absorción de los elementos (Hernández et al. 1996). Según Fageria et al. (2010), los efectos positivos, en cuanto a la aplicación de fósforo en fríjol común y en la asociación haba-maíz, los atribuyen, principalmente, al incrementó en el desarrollo del sistema radical, con lo que se mejora la toma de nutrientes minerales. 


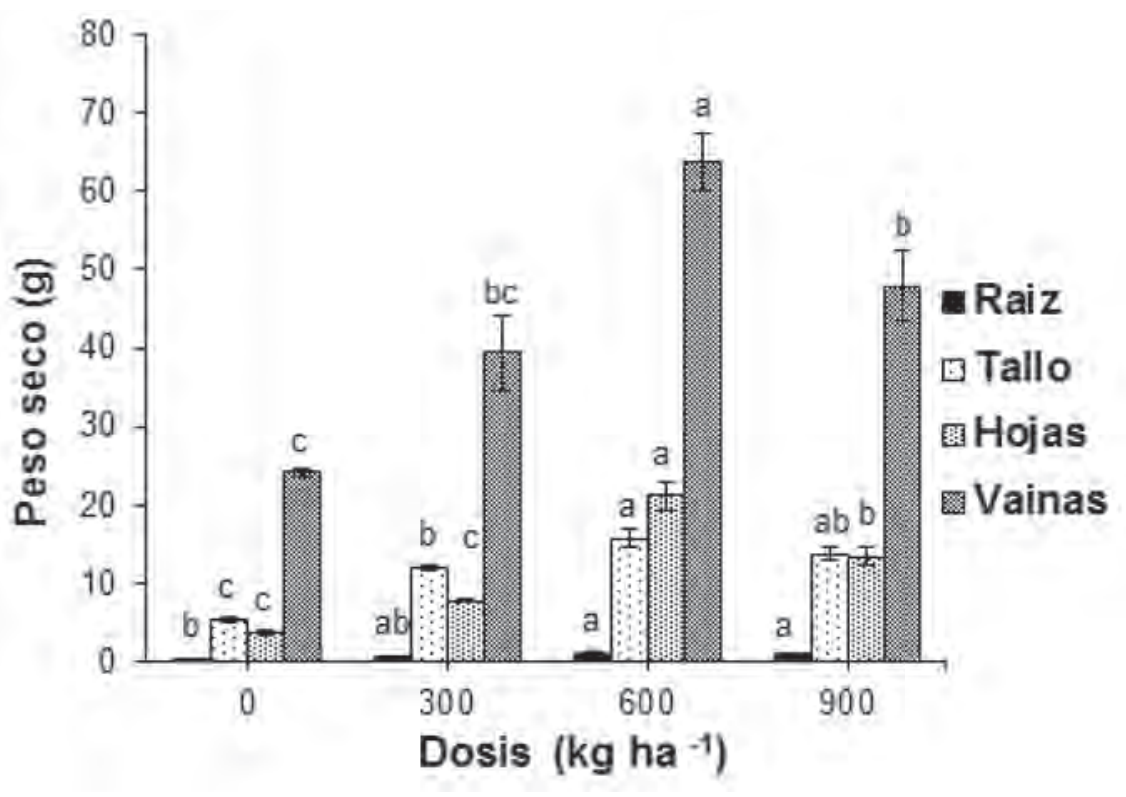

Figura 2. Peso seco de órganos en fríjol (Phaseolus vulgaris L.) cv 'ICA Cerinza', sometido a diferentes dosis de fosfato térmico. Tratamientos seguidos de letras distintas presentan diferencias significativas, según la prueba de Tukey $(P \leq 0,05)$. Barras verticales indican error estándar $(n=4)$.

Las plantas con deficiencia de fósforo crecen lentamente, sus hojas se tornan verde oscuras con áreas rojizas y presentan tallos delgados (Rojas, 1993), disminuye la captación de radiación solar por el cultivo y la eficiencia de conversión de la radiación interceptada en materia seca, produciendo, por lo tanto, mermas en la materia seca total.

Según Morales et al. (2007), en fríjol, cuando se adicionaron $60 \mathrm{~kg} \mathrm{P}_{2} \mathrm{O}_{5}$ ha $^{-1}$, se obtuvo una producción de materia seca superior al testigo en $27 \%$; ello, se puede atribuir a que este nutriente junto con el nitrógeno están relacionados directamente con el contenido de clorofila y el tamaño del dosel vegetal, lo cual, aumenta la intercepción de radiación solar y fotosíntesis, generando una mayor acumulación de materia seca (Abayomi et al. 2008). En caupi, el peso seco de las raíces y la materia seca total mostraron resultados positivos en respuesta a la aplicación de fósforo (Okeleye \& Okelana, 2000; Nkaa et al. 2014).

Hernández et al. (1996) indican que aplicaciones según requerimientos nutricionales de fósforo en fríjol incrementan la acumulación de masa seca en raíces, en tallos, en hojas, en peciolos y en vainas. Por su parte, Medina et al. (1999) indican que el fósforo juega un papel importante en la composición de las membranas y estimula el crecimiento; Haruna \& Aliyu (2011) señalan que inicia la formación de nódulos e influencia la eficiencia de la simbiosis Rhizobium leguminosa, que es un proceso de conducción de energía y ello con- tribuye al aumento de materia seca en los distintos órganos de la planta (Nkaa et al. 2014).

Por su parte, el área foliar mostró diferencias significativas entre tratamientos, según la prueba de Tukey $(P \leq 0,05)$. El tratamiento de $600 \mathrm{~kg} \mathrm{ha}^{-1}$ de fosfato térmico presentó el mayor valor de área foliar, con $1750,32 \pm 81,39 \mathrm{~cm}^{2}$, sin diferencias estadísticas con el tratamiento de $900 \mathrm{~kg} \mathrm{ha}^{-1}$, pero sí con la dosis de $300 \mathrm{~kg} \mathrm{ha}^{-1}$ y el testigo, expresó el menor valor, con $855,13 \pm 13,25 \mathrm{~cm}^{2}$ (Figura 3).

Varios estudios fisiológicos indican que el área foliar está estrechamente relacionado con la dinámica de la acumulación de materia seca, fósforo y otros nutrientes (Marschner, 2012), definiéndose a la lámina foliar, como la parte más importante del aparato fotosintético de la planta (Pineda-Mares et al. 2001); lo anterior está de acuerdo con lo observado en el presente estudio.

En cuanto a las variables de producción, se encontraron diferencias significativas entre tratamientos, para número de vainas por planta y rendimiento. El mayor valor fue de $21 \pm 1,08$ y $2326,6 \pm 184,68 \mathrm{~kg} \mathrm{ha}^{-1}$, respectivamente, observado, en el tratamiento de $600 \mathrm{~kg}$ de fosfato térmico, ha ${ }^{-1}$. Para las variables de número de granos por vaina y por peso de cien granos, no se presentaron diferencias estadísticas entre tratamientos (Tabla 2). 


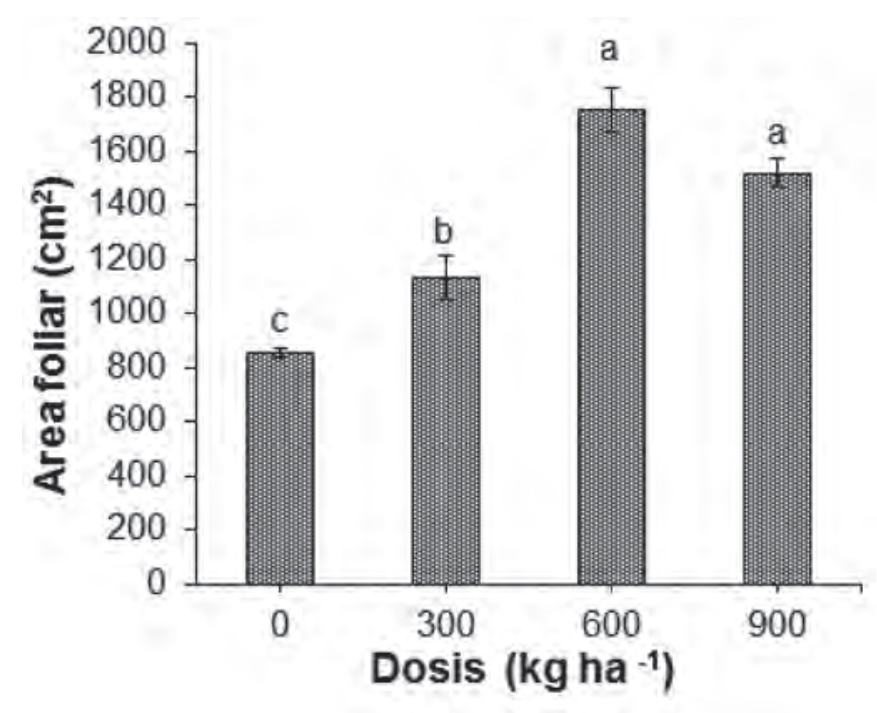

Figura 3. Área foliar en fríjol (Phaseolus vulgaris L.) cv 'ICA Cerinza', sometido a diferentes dosis de fosfato térmico. Tratamientos seguidos de letras distintas presentan diferencias significativas, según la prueba de Tukey $(P \leq 0,05)$. Barras verticales indican error estándar $(n=4)$.

Tabla 2. Efecto de diferentes dosis de un fosfato térmico sobre componentes de rendimiento en fríjol cv 'ICA Cerinza', en condiciones agroecológicas de Tunja, Boyacá.

\begin{tabular}{|c|c|c|c|c|}
\hline $\begin{array}{l}\text { Tratamientos } \\
\left(\mathrm{kg} \mathrm{ha}^{-1}\right)\end{array}$ & NVP & NGV & P100 & $\begin{array}{l}\text { Rendimiento } \\
\left(\mathrm{kg} \mathrm{ha}^{-1}\right)\end{array}$ \\
\hline 0 & $12 \pm 0,4 \quad b$ & $3,35 \pm 0,14 a$ & $63,11 \pm 0,73$ a & $1408,7 \pm 75,5 b$ \\
\hline 300 & $15,5 \pm 2,02 \mathrm{ab}$ & $3,14 \pm 0,11$ a & $66,37 \pm 1,51$ a & $1781,1 \pm 199,0 \mathrm{ab}$ \\
\hline 600 & $21 \pm 1,08$ a & $3,09 \pm 0,12 a$ & $64,22 \pm 0,53 a$ & $2326,6 \pm 184,6 a$ \\
\hline 900 & $20 \pm 1,77$ a & $3,17 \pm 0,02$ a & $61,99 \pm 1,36 a$ & $2192,5 \pm 226,6 a$ \\
\hline
\end{tabular}

Promedios con letras distintas en la misma columna indican diferencia significativa, según la Prueba de Tukey $(\mathrm{P} \leq 0,05)$. Media acompañada del error estándar $( \pm)$. NVP: Número de vainas por planta; NGV: Número de granos por vaina; P100: Peso de 100 granos al 14\% de humedad.

Según los resultados, se pudo observar que el componente de rendimiento que determinó en mayor proporción el rendimiento final en $\mathrm{kg} \mathrm{ha}^{-1}$ fue el número de vainas por planta, ya que se observó un aumento en comparación con el testigo, mientras que las demás variables fueron similares. El número de vainas por planta, a menudo, es recomendado como un criterio de selección indirecta para aumentar la productividad, principalmente, debido a su mayor y más consistente correlación con el rendimiento (Fageria et al. 2010).

También, se pudo observar que, a medida que el número de vainas aumentó, el número de granos por vaina disminuyó, posiblemente, debido a un efecto de dilución por crecimien- to. En cuanto al aumento de peso de cien granos, se dio el mejor resultado numéricamente con la aplicación de $300 \mathrm{~kg}$ de fosfato térmico ha ${ }^{-1}$, que se podría atribuir a la función del fósforo en la formación de semillas y llenado de grano acumulado, como fitatos (Haruna, 2011; Taiz \& Zeiger, 2010).

Según investigaciones realizadas la aplicación de $150 \mathrm{~kg} \mathrm{ha}^{-1}$ de fósforo junto con aplicación de nitrógeno logró aumentar la biomasa total, el número de vainas, el número de granos, el rendimiento de grano y la eficiencia en el uso del agua (Apáez et al. 2013). Otros autores afirman que el fósforo desempeña un papel importante en la fotosíntesis, la respiración, el almacenamiento, la transferencia de energía, 
la división y el crecimiento celular, promoviendo, además, la rápida formación y crecimiento de las raíces y mejorando la calidad de granos (Múnera \& Meza, 2012).

En cuanto a la variable concentración de fósforo en tejido foliar al momento de maduración, se presentaron diferencias significativas entre tratamientos $(\mathrm{P} \leq 0,05)$. La mayor concentración de $\mathrm{P}$ se obtuvo con la dosis de aplicación de $600 \mathrm{~kg}$ de fosfato térmico ha ${ }^{-1}$, con un valor de $0,32 \pm 0,01 \%$, sin diferencias estadísticas con la mayor dosis aplicada, pero sí con $300 \mathrm{~kg} \mathrm{ha}^{-1}$ y el testigo que presentó la menor concentración con 0,21 $\pm 0,004 \%$ al terminar la etapa de llenado de vainas (Figura 4). Estos resultados indican que el uso de un fosfato térmico contribuyó al aumento de la concentración en tejido de fósforo, representado en un incremento en el crecimiento y en la productividad de fríjol cv 'ICA Cerinza', ya que se observó una relación directa con variables, como contenido de clorofila, masa seca de órganos, área foliar y número de vainas por planta.

También, el fosfato térmico, al tener concentraciones de silicio, los sitios de fijación de $\mathrm{P}$ pueden ser ocupados parcialmente por el ácido silícico y en contraste cuando el $\mathrm{P}$, se adsorbe, el si se libera a la solución del suelo; estas reacciones de adsorción-desorción son reversibles y constituyen un mecanismo fundamental para mejorar la disponibilidad de $P$ en los suelos, generando una buena dinámica nutricional (Datnoff et al. 2007). El mismo autor indica que, en el suelo, uno de los efectos más importantes de la aplicación de enmiendas silicatadas es mejorar la disponibilidad de P y así mejorar su absorción por las plantas; estos efectos son muy evidentes en suelos tropicales, debido a la competencia del ion $\mathrm{H}_{2} \mathrm{PO}_{4}$ y el $\mathrm{H}_{4} \mathrm{SiO}_{4}$, por sitios de adsorción sobre la superficie de arcillas y de óxidos e hidróxidos de Fe y Al.

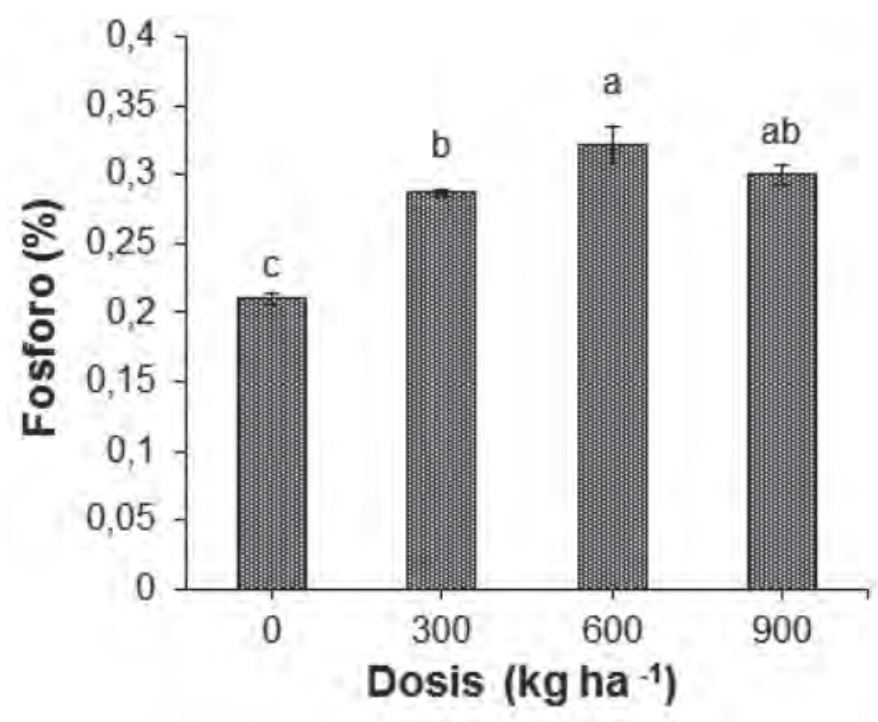

Figura 4. Concentración en tejido vegetal de fósforo (\%) en fríjol (Phaseolus vulgaris L.) cv 'ICA Cerinza', sometido a diferentes dosis de fosfato térmico. Tratamientos seguidos de letras distintas presentan diferencias significativas, según la prueba de Tukey $(P \leq 0,05)$. Barras verticales indican error estándar $(n=4)$.

Según Marschner (2012), en la mayoría de plantas la concentración de fósforo que se considera adecuada es 0,2\% en relación al peso seco, en el presente estudio, se tuvo una mayor acumulación de fósforo a nivel foliar, que tiene un efecto directo en la capacidad fotosintética de las plantas (Singh et al. 2013). Otros afirman que el fósforo es acumulado en partes vegetativas hasta la floración, para luego ser movilizado hacia los granos en crecimiento; en maíz, se han encontrado concentraciones de $0,17 \%$ y $0,22 \%$ peso seco y en algodón, valores de 0,24\% y 0,66\%, para hojas y grano, respectivamente (Múnera \& Meza, 2012).
Se puede concluir, que la aplicación de T-Fos ${ }^{\circledR}$ (fosfato térmico) en condiciones de suelo, moderadamente ácidas, con pH 5,53, favoreció de manera significativa variables, como contenido total de clorofila, masa seca de órganos, área foliar, fósforo en tejido foliar y número de vainas por planta. Además, la aplicación del fosfato térmico frente al testigo generó un aumento de un $65 \%$, en el rendimiento con la dosis de $600 \mathrm{~kg} \mathrm{ha}^{-1}$.

Los resultados encontrados en la presente investigación indican que la aplicación de fosfato térmico enriquecido con cal- 
cio, silicio y magnesio contribuye a aumentar el rendimiento de fríjol, en un suelo de condición moderadamente ácida, haciendo más eficiente la absorción de fósforo, posiblemente, debido a las características del producto que mejoran la solubilidad en condiciones ácidas, siendo una alternativa importante en la incorporación de las prácticas agronómicas.

Agradecimientos: La presente investigación se realizó con el apoyo de Cales y Derivados Calcáreos Rio Claro Naranjo y Compañía S.C.A. y la Universidad Pedagógica y Tecnológica de Colombia, sede Tunja. Conflicto de intereses: El manuscrito fue preparado y revisado con la participación de todos los autores, quienes declaramos que no existe conflicto de intereses que ponga en riesgo la validez de los resultados presentados.

\section{BIBLIOGRAFÍA}

1. ABAYOMI, Y.A.; AJIBADE, T.; SAMMUEL, O.; SAADUDEEN, B. 2008. Growth and yield responses of cowpea (Vigna unguiculata L.) genotypes to nitrogen fertilizer (NPK) application in the Southern Guinea Savanna zone of Nigeria. Asian J. Plant Sci. 7:170176.

2. ADATIA, M.; BESFORD, R. 1986. The effects of silicon on cucumber plants grown in recirculating nutrient solution. Ann. Bot. 58:343-351.

3. APÁEZ, P.; ESCALANTE, J.; RAMÍREZ, P.; DOUGLAS, S.; SOSA, E.; OLALDE, V. 2013. Eficiencia agronómica de nitrógeno y fósforo en la producción de fríjol chino en espaldera de maíz. Terra Latinoamericana. 31(4):285-293.

4. ARIAS, J.H.; JARAMILLO, M.; RENGIFO, T. 2007. Manual Técnico: Buenas Prácticas Agrícolas (BPA) en la producción de fríjol voluble. CORPOICA - MANA - FAO. C.I. La Selva. Medellín. 168p.

5. AZCÓN-BIETO, J.; TALÓN, M. 2013. Fundamentos de fisiología vegetal. $2^{a}$ ed. Mc Graw Hill. Universidad de Barcelona. España. 656p.

6. BEEBE, S.; RAO, I.; CAJIAO, C.; GRAJALES, M. 2008. Selection for drought resistance in common bean also improves yield in phosphorus limited and favorable environments. Crop Sci. 48:582-592.

7. BROUGHTON, W.; HERNANDEZ, G.; BLAIR, M.; BEEBE, S.; GEPTS, P.; VANDERLEYDEN, J. 2003. Beans (Phaseolus spp.) - model food legumes. Plant and Soil. 252:55-128.
8. CAKMAK, I.; HENGELER, C.; MARSCHNER, H. 1994. Partitioning of shoot and root dry matter and carbohydrates in bean plants suffering from phosphorus, potassium and magnesium deficiency. J. Exp. Bot. 45:1245-1250.

9. CAKMAK, I; KIRKBY, E.A. 2008. Role of magnesium in carbon partitioning and alleviating photooxidative damage. Physiol. Plant. 133:692-704.

10. CASTRO, H. 1998. Fundamentos para el conocimiento y manejo de suelos agrícolas: Manual técnico. Instituto Universitario Juan de Castellanos. 360p.

11. CORDELL, D.; DRANGERT, J.; WHITE, S. 2009. The story of phosphorus: global food security and food for thought. Global Environ Change. 19:292-305.

12. DATNOFF, L.; RODRIGUES, F.; SEEBOLD K. 2007. Silicon and Plant Disease. En: Mineral Nutrition and Plant Disease: The American Phytopathological Society. p.233-246.

13. FAGERIA, N.K.; BALIGAR, V.C.; MOREIRA, A.; PORTES, T. 2010. Dry bean genotypes evaluation for growth, yield components and phosphorus use efficiency. J. Plant Nutr. 33:2167-2181.

14. FENALCE. 2015. Federación Nacional de Cultivadores de Cereales y Leguminosas. Departamento económico y apoyo a la comercialización. Indicadores cerealistas. Bogotá D.C. 102p.

15. FERNÁNDEZ, M. 2007. Fósforo: amigo o enemigo. ICIDCA. Sobre los derivados de la caña de azúcar. 41(2):51-57.

16. FERNÁNDEZ, S.; NOGUERA, R. 2003. Producción de fosfatos térmicos a partir de rocas fosfóricas nacionales. Agr. Trop. 53(1):49-58.

17. GILBERT, N. 2009. The disappearing nutrient. Nature.461:716-718.

18. HARUNA, I. 2011. Dry matter partititioning and grain yield potential in sesame (Sesamum indicum L.) as influenced by poultry manure, nitrogen and phosphorus at Samaru, Nigeria. J. Agric. Technol. 7:15711577.

19. HARUNA, I.; ALIYU, L. 2011. Yield and economic returns of sesame (Sesamum indicum L.) as influenced by poultry manure, nitrogen and phosphorus at Samaru, Nigeria. Elixir Agric. 39:4884-4887. 
20. HERNÁNDEZ, G.; TOSCANO, V.; MÉNDEZ, N.; GÓMEZ, L.; MULLINGS, M. 1996. Efecto de la concentración de fósforo sobre su asimilación en tres genotipos de fríjol común (Phaseolus vulgaris L.). Agron. Mesoam. 7(1):80-85.

21. MARSCHNER, P. 2012. Mineral nutrition of higher plants. $3^{\text {rd }}$. Ed. Elsevier. Oxford, UK. 645p.

22. MEDINA, G.; OROZCO, M.; BOLÍVAR, J.; RAMÍREZ, P. 1999. Acumulación y concentración de nitrógeno, fósforo y potasio en Gypsophila paniculata L. cv. Perfecta. Agron. Colomb. 16(1-3):46-50.

23. MORALES, E.; ESCALANTE, J.; LÓPEZ, J. 2007. Producción de biomasa y rendimiento de semilla en la asociación girasol (Helianthus annuus L.) fríjol (Phaseolus vulgaris L.) en función del nitrógeno y fósforo. Ciencia Ergo Sum. 14(2):177-183.

24. MÚNERA, G.; MEZA, D. 2012. El fósforo elemento indispensable para la vida vegetal. Manual. Universidad Tecnológica de Pereira. 52p.

25. NKAA, F.A.; NWOKEOCHA, O.; IHUOMA, O. 2014. Effect of phosphorus fertilizer on growth and yield of cowpea (Vigna unguiculata). J. Pharmacy Biological Sc. 9(5):74-82.

26. OKELEYE, K.A.; OKELANA, M. 2000. Effect of phosphorus fertilizer on nodulation, growth, and yield of cowpea (Vigna unguiculata) varieties. Indian J. of Agric. Sci. 67(1):10-12.

27. PINEDA-MARES, P.; MARTÍNEZ-MONTOYA, J.; AMANTE-OROZCO, A.; RUIZ-VERA, V. 2001. Respuesta del maíz al fósforo y un mejorador de suelos en áreas yesosas de la zona media de San Luis de Potosí. Rev. Chapingo Serie zonas áridas. p.106-113.
28. ROJAS, M. 1993. Fisiología vegetal aplicada. Interamericana. Mc Graw Hill. México. 275p.

29. SHEKARI, F.; ABBASI, A.; MUSTAFAVI, S. 2015. Effect of silicon and selenium on enzymatic changes and productivity of dill in saline condition. J. Saudi Soc. Agr. Sc. Corrected Proof. In Press. http://dx.doi. org/10.1016/j.jssas.2015.11.006.

30. SINGH, S.K.; REDDY, V.R. 2015. Response of carbon assimilation and chlorophyll fluorescence to soybean leaf phosphorus across $\mathrm{CO}_{2}$ : Alternative electron sink, nutrient efficiency and critical concentration. J. Photochem. Photobiol. B: Biology.151:276-284.

31. SINGH, S.K.; BADGUJAR, G.B.; REDDY, V.R.; FLEISHER, D.H.; TIMLIN, D.J. 2013. Effect of phosphorus nutrition on growth and physiology of cotton under ambient and elevated carbon dioxide. J. Agron. Crop Sci. 199:436-448.

32. TAIZ, L; ZEIGER, E. 2010. Plant Physiology. $5^{\text {th }}$ Ed. Sinauer Associates, Sunderland. 782p.

33. ULLOA, J.A.; ROSAS ULLOA, P.; RAMÍREZ, J.C.; ULLOA, B.E. 2011. El fríjol (Phaseolus vulgaris): su importancia nutricional y como fuente de fitoquímicos. Rev. Fuente Año 3(8):5-9.

34. ZAPATA, E.; ROY, R. 2007. Utilización de las rocas fosfóricas para una agricultura sostenible. Boletín FAO. Fertilizantes y nutrición vegetal 13. Roma. 177p.

Recibido: Septiembre 15 de 2016

Aceptado: Febrero 16 de 2017

Cómo citar:

Quintana-Blanco, W.A.; Pinzón Sandoval, E.H.; Torres, D.F. 2017. Efecto de un fosfato térmico sobre el crecimiento y producción de fríjol (Phaseolus vulgaris L.) cv Ica Cerinza. Rev. U.D.C.A Act. \& Div. Cient. 20(1): 51-59. 